\title{
Space Frame Optimisation with Spectral Clustering
}

\author{
Xinwei Zhuang and Sean Hanna
}

\begin{abstract}
This paper borrows the concept of spectral clustering in the computer vision field, proposes an alternative approach to optimise space frame structure. Spectral clustering was implemented to segment the whole structure into two subclusters. Then genetic algorithm was used to optimise member sizes of each subcluster separately. It is hypothesized that optimizing the structural stability for subassemblies will largely reduce the search space, which allows greater computational efficiency. The program has been developed in MATLAB and tested on differently shaped space frame structure under varied loading conditions. Results show that for a heterogeneous structure with high a level of complexity, the implementation of spectral clustering can separate the enormous search space of GA down to smaller search space, leading to faster convergence with increased the computational efficiency, while providing an equivalent or better optimisation solution.
\end{abstract}

Index Terms-Computational efficiency, genetic algorithm, space frame structure, spectral clustering, structural optimization.

\section{INTRODUCTION}

Genetic algorithm has long been used as a global optimum searching tool for structural optimisation. Different aspects of structural optimisation by genetic algorithm for skeletal structures such as sizing, topology and layout have been studied comprehensively (Shrestha and Ghaboussi [1]). Since space frame structures of unprecedented scale have been used in a wide range from aircraft to architecture and art, the optimisation problems of structures can be computationally inefficient when encountering a complex structure with too many variables to optimise. The growth rates of genetic algorithm for time complexity are mostly described as exponential with the addition of every new variable (Mange and Tomassini [2]). When solving the problems with a large number of variables, the search space can be considerably big, such that GA will lose efficiency and require a long time to converge (Rylander and Foster [3]).

Simon [4], [5] suggested that hierarchical system will evolve more efficiently than non-hierarchical systems. When a system includes substructures interrelated to each other, this hierarchic system produced by independent subsystem has a potential for rapid evolution. When the substructures are nearly independent from each other, this system is nearly decomposable. A nearly-decomposable system has the

Manuscript received August 25, 2019; revised April 12, 2020.

Xinwei Zhuang was with University College London, London, WC1E 6BT UK (e-mail: ucqbxz9@ucl.ac.uk).

Sean Hanna is with University College London, London, WC1E 6BT UK (e-mail: s.hanna@ucl.ac.uk). corresponding genes which can be supposed to operate nearly independently, and hierarchically control the phenotype of specific organs, and the time required for the evolution of a complex form depends critically on the number and distribution of the subassemblies, the systems that evolve by assembly of simpler systems evolves faster than non-hierarchical systems without subassemblies even of comparable size. Building upon Simon, when optimizing a complex structure becomes computationally infeasible for a considerable number of members, one way to improve computational efficiency is to reduce the size of search space according to its hierarchy and decomposability, and optimizing its structural stability for its subassemblies rather than a whole.

It is proposed that reducing the dimension of the search space would result in a much more efficient optimisation with GA. The expected magnitude of reduction in time depends on the complexity of genetic algorithm (Pelikan and Lobo [6], He and Yao [7]). Rudolph [8] proved that the computational complexity for genetic algorithm can be attributed to the most time-consuming part, fitness evaluation. For space frame optimisation, fitness is evaluated via finite element approach, whose big $\mathrm{O}$ notation is $O\left(N W^{2}\right)$, where $\mathrm{N}$ is the number of nodes of the structure and $\mathrm{W}$ is the bandwidth of the banded stiffness matrix [9]. The stiffness matrix is the number of nodes multiplied by the degree of freedom per node. Thus, the big $O$ notation of genetic algorithm is simplified to $O\left(N^{3}\right)$, where $N$ denotes the number of nodes. The complexity of the optimisation problem will decrease drastically as the number of nodes decreases, as proportional to the cube of $N$. As in equation (1), were the total number of nodes reduced by half, the optimisation algorithm theoretically would require only one-fourth of the original time.

$$
O\left(\left(\frac{N}{2}\right)^{3}+\left(\frac{N}{2}\right)^{3}\right) \approx O\left(\left(\frac{N}{4}\right)^{3}\right) \leq O(N)
$$

Clustering algorithm is used to decompose large data sets, among which spectral clustering regards the data points as vertexes and the similarity between all pairs of data points as weighted edges, transforming the clustering problem into a graph partition problem (Von Luxburg [10]). Spectral clustering has proven efficient in splitting data hierarchically in complex systems (Queyroi [11]). Its applications have been fruitful and percolated to disciplines including statistics, computer vision, social science, computational biology, and, recently, architecture, (Queyroi [11], Shi and Malik [12], Ng [13], Hanna [14]), but few studies are known to use it to partition a structure for structural analysis.

In this paper, we test whether a space frame structure is more efficiently optimized as a whole, or as separate, smaller parts defined by clustering. The space frame structure is viewed as a connected, evenly weighted graph, in which the 
tubular members connecting two nodes are regarded as the edges between vertices, and their size as the weight of the edges. We decompose the complex structure into parts by spectral clustering, thus reducing the input instances by roughly half of the original. We then optimise for each subassembly separately and test for computational efficiency. Different shapes of the space frame are compared. Any increase in efficiency will corroborate the hypotheses that space frame structures of that type are separable for structural optimisation, and that the implantation of spectral clustering can reduce the computation time according to the computational complexity. The process of the modified optimisation algorithm is demonstrated in Fig. 1.

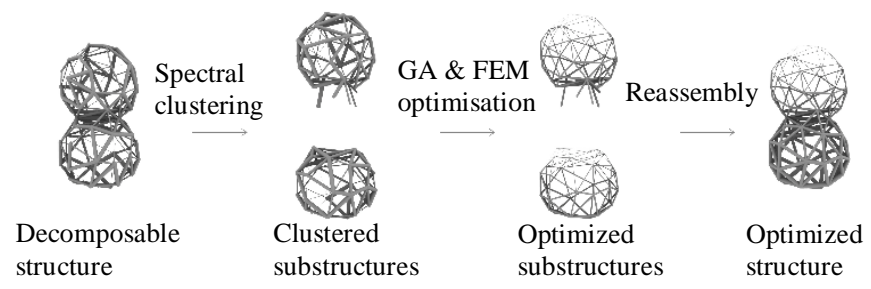

Fig. 1. Illustration of the proposed optimisation process.

\section{MethodolOGY}

Experiments are conducted to testify the hypothesis that whether the implementation of the clustering algorithm can improve the computational efficiency of the optimisation. The selected structures are vertically splittable but have distinct decomposability. Fig. 2 shows the two space frame structures used in the experiments. Varieties include geometries, densities (number of nodes) and loading conditions. In the experiments, structures are subjected to a vertical uniform load simulating self-weight and a lateral uniform load representing wind.

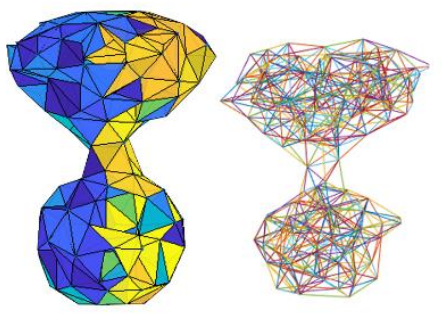

(a)

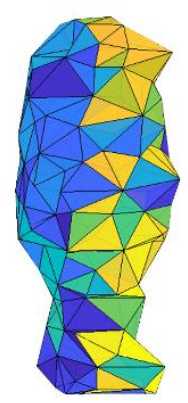

(b)

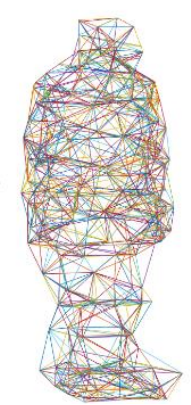

Fig. 2. Delaunay triangulated structure and connections (a) gross structure (b) human figure.

\section{A. Spectral Clustering}

Spectral clustering is used to partition the structure for its ability to process arbitrarily shaped data without making assumptions about the form of the cluster, and its robustness, converging to the global optimum without being oversensitive to the outliers (Xu and Tian [15]).

The triangulated space frame structure is regarded as a graph, in which the nodes are deemed as the input vertices, whereas the steel members represent the connections between two vertices, i.e., the adjacency. Spectral clustering segments vertices based on their affinities. Regarding the adjacencies between all pairs of data points as weighted edges, the goal is to perform inherently hierarchical graph partitioning, where the weight of the connections between different subparts is as small as possible, and the total weight of the connections within the same group is as high as possible (Von Luxburg [10], Shi and Malik [12]). Spectral clustering algorithm will split the structure into two substructures with a cutting plane will cause minimal damage to the original structure. Fig. 3 illustrates the clustering result. Spectral clustering clearly separates the structure by a hyperplane that has a minimal effect on the topology. For structures with different geometrical decomposability, substructures can have distinct ratios. The unequal partitioning guarantees minimal damage but is later found to affect the computation efficiency improvement in the optimisation process.

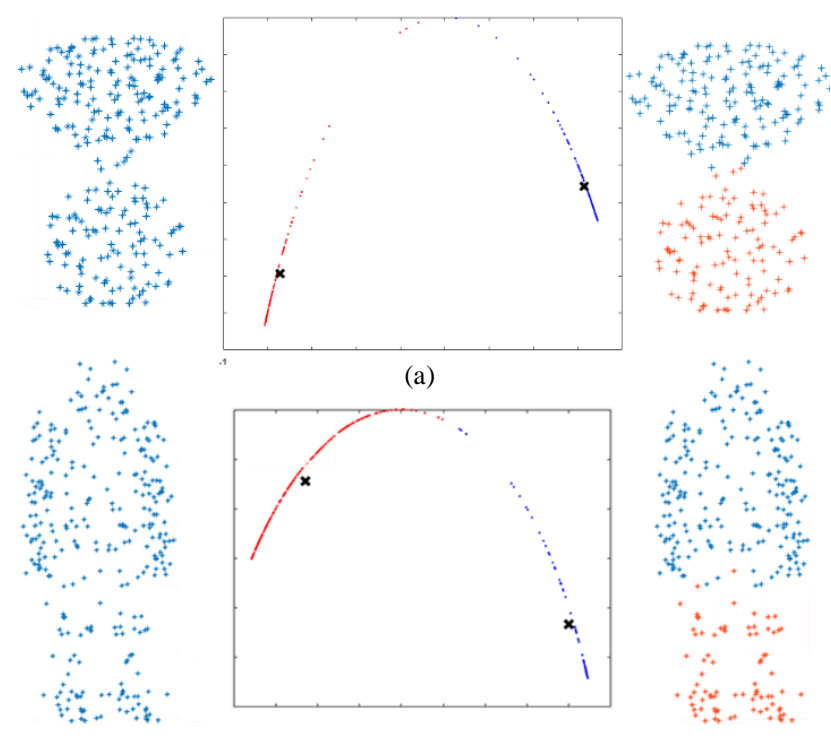

(b)

Fig. 3. Spectral clustering result for (a) dense gross geometry, (b) human figure-like structure. Left: initial point clouds; middle: Laplacian eigenmap: the presentation of clustered data points and centroids after unnormalized Laplacian mapping; right: the clustering result back into 3D space in two clusters.

\section{B. Optimisation with Genetic Algorithm and Finite Element Analysis}

Genetic algorithm was used as a search tool to find the optimal combination of member sizes to minimize deflection of the space frame structure. In this project, three genes together define the radius for the section of the steel member. The member sizes are encoded in 3-bit binary code from 000 to 111 , representing from 0.1 to 0.8 , which are then translated to the phenotype, controlling the section radii of the members. The chromosome length is determined by the number of members. Genetic algorithm uses fitness proportionate selection to select two individuals from the last generation as parents and randomly chooses one point in chromosome for crossover. A $3 \%$ mutation for each allele avoids optimization falling into local optima. The genes are decoded from binary code to the corresponding real numbers, which are used as the input for FEA, where the stability of each structure is evaluated and fed back to GA as the fitness value. Finally, the population is sorted, and the best individual in the current generation is recorded. Individuals with greater deflection have less possibility of being chosen as a parent for the next 
generation. The experiments terminate when the population has evolved through a maximum generation or when the maximum computation time is reached. Random seed is used to initialize the first population for the two optimisation processes using the same set of genes with an identical initiation.

In the general optimisation, to minimize the deflections, the upper structure is always lighter, whereas the lower structure is always thicker. However, in the separated optimisation, FEA fails to analyze the whole picture between two substructures. A rationalization of the ratio of the two amounts of steel is required. A scale coefficient for the radii between two clusters is tested, and a plot of the average of the scaling factor versus deflection for ten experiments is shown in Fig. 4. The scale coefficient varies among different structures according to their geometry. The optimum scaling factor of 1.4 for the gross structure, and 1.7 for a human-figured structure is selected because a further increase of the scaling factor does not substantially minimize the deflection but leads to a waste of material.

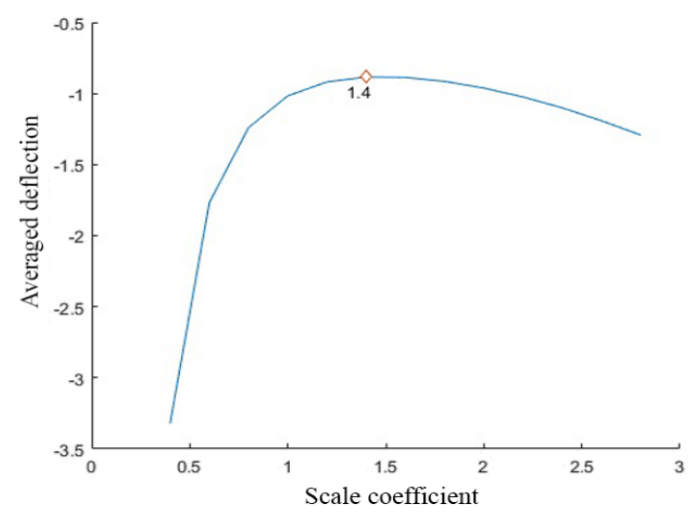

(a)

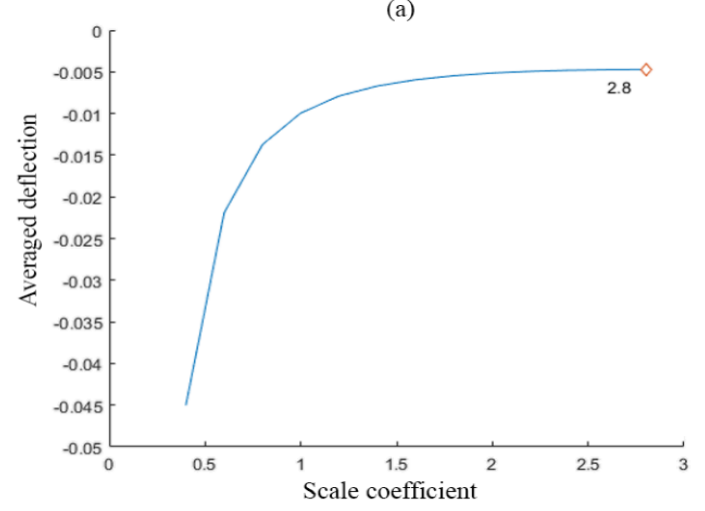

(b)

Fig. 4. The scale factor for (a) structure with two-gross geometries; (b) human-figured structure.

\section{RESULtS}

\section{A. Results for the Clearly Clustered Structure}

The first set of experiments are conducted for the two-gross geometry shown in Fig. 3(a) under two loading conditions: gravity and wind load. The deflections and the corresponding time for two optimisation processes are shown in Fig. 5. To ameliorate the effect of randomness, each result presented below is a diagram plotted from the average data for three experiments with identical initiation.

The deflection of the separated optimisation rises intensely at the beginning, followed by slight fluctuation, and reaches a plateau in the end. The whole optimisation experiences a more moderate rise. The separated optimisation requires only $41 \%$ of the time needed for the whole-structure optimisation to converge and stabilize at a $20.5 \%$ better fitness value. The efficiency of the optimisation algorithm overtime is computed as the deflection improvement within the time interval of the execution time required for one run. The increase of fitness of the separate cluster optimisation is around 1.5 times higher than that for the whole-structure optimisation in the first ten runs, and gradually decreases down to 0 with vibrations, whereas the effectiveness of the whole-structure optimisation is only significant in the beginning and quickly drops to 0 in successive generations. After the first ten runs, although considerable oscillations are observed from negative to positive, the fluctuation inefficiency does not change the leading position of the segmented optimisation in fitness.

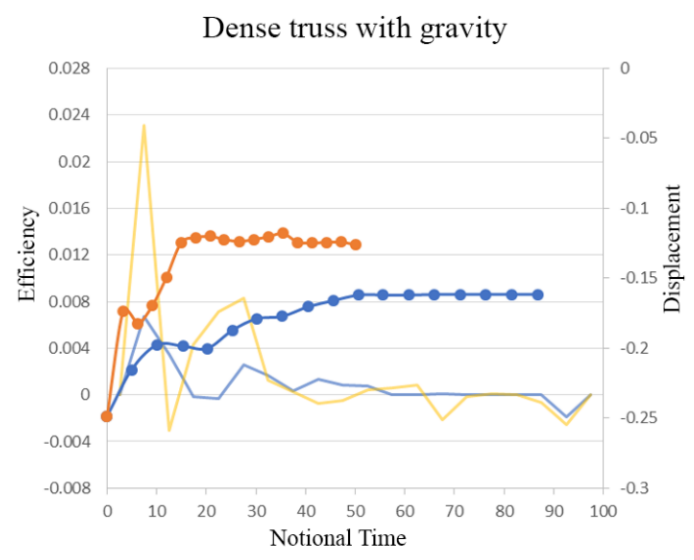

Dense truss with gravity \& wind load

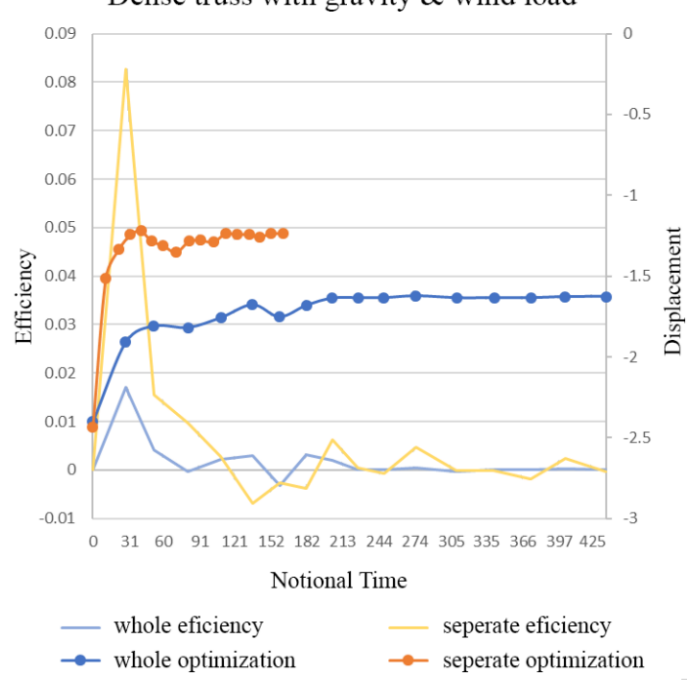

Fig. 5. Result with gravity and wind load for the gross structure with high density.

Another experiment for the same geometry but with a low density of nodes is conducted to examine whether a different level of complexity has an influence on the computation efficiency (Fig. 6). For the structure with 69 nodes and 181 members, the segmented optimisation takes more time than the whole-structure optimisation to complete 50 iterations and provide a fitness no higher than the whole optimisation. Besides, the oscillation in efficiency and the resulting fluctuation in fitness are considerable compared to that of the whole-structure optimisation. 
Loose truss with gravity

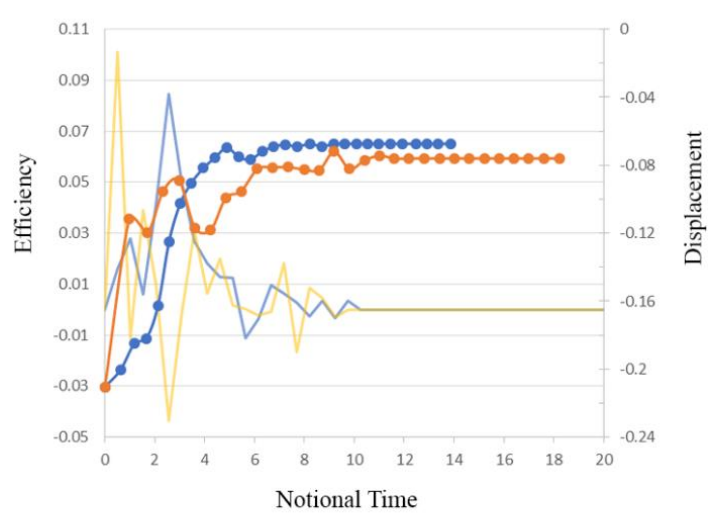

Loose truss with gravity \& wind load

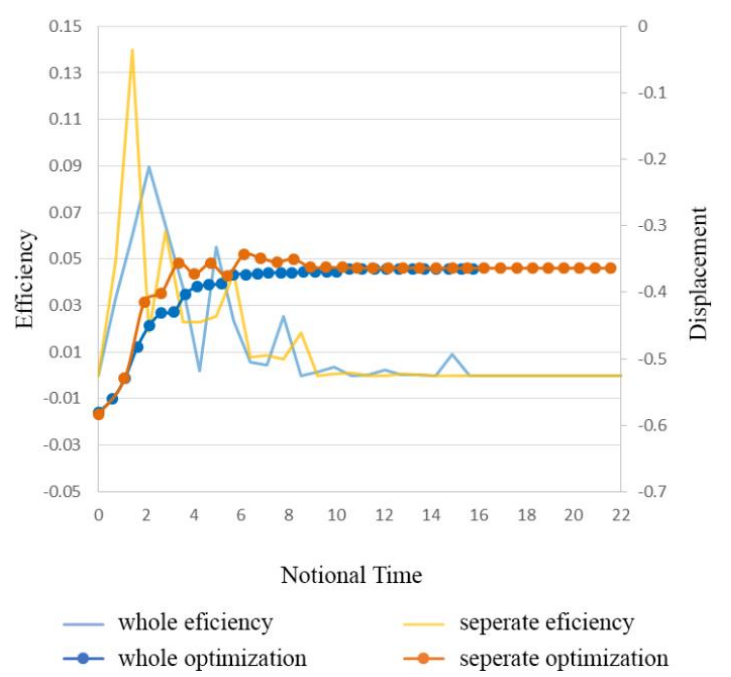

Fig. 6. Result with gravity and wind load for gross truss structure with low density.

\section{B. Results for a Human-Figure-Like Structure}

A space frame structure modified from a human figure model (Fig. 3(b)) without implicit clusters is also tested to examine the influence of topology on efficiency. Results are shown in Fig. 7.

The uneven clustering makes the computation time not reducible as much as when the structure is equally separated. Although the efficiency of the segmented optimisation is approximately ten times greater than that of the whole-structure optimisation in the first ten runs, it oscillates too fierce to hold an optimal deflection in the subsequent runs The initial effectiveness of the whole optimisation is much lower than that of the segmented one, but few negative values are observed among all generations, constituting a smooth increase in fitness during the evolution. The whole-structure optimisation reaches a slightly higher fitness in the end, requiring a longer time, but presents an increasing trend till the last run.

The optimisation process under gravity and lateral load presents a similar result. The separated-structure optimisation achieved a minimal deflection $23.8 \%$ higher than the whole-structure optimisation requiring only one-third of the time for the whole. Though the general deflection is higher due to the displacement in the horizontal axis by the lateral load, no obvious influences from the different load conditions were observed either on the fitness level or on the computational time.

Human figure structure with gravity

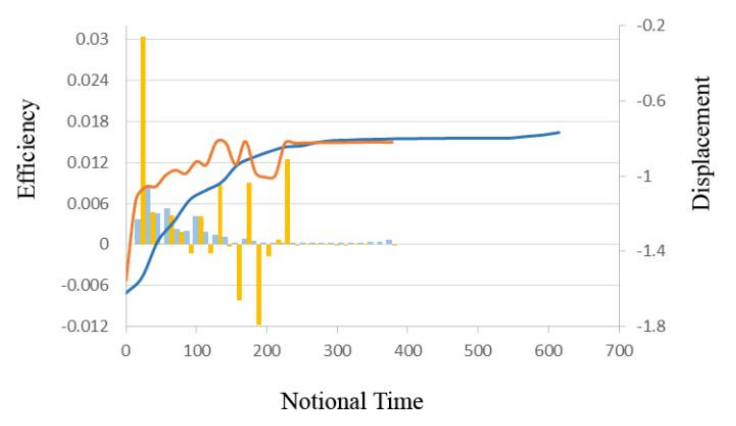

Human figure structure with gravity \& wind load

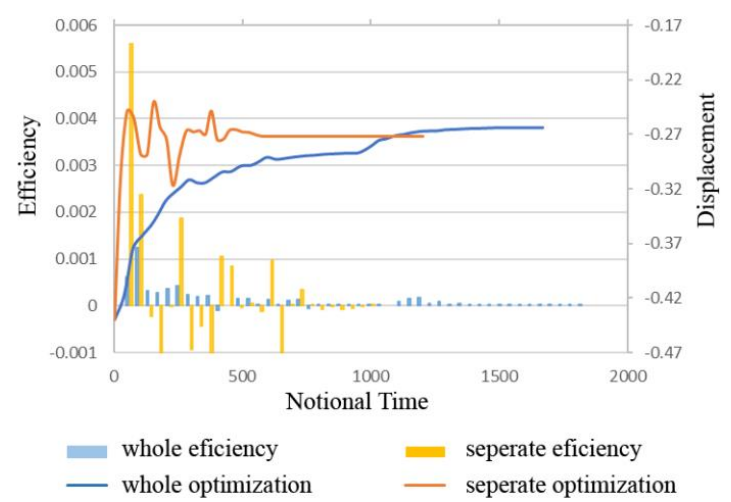

Fig. 7. Result with gravity left and wind load for a human figure-like-structure.

\section{Discussion}

According to the big $\mathrm{O}$ notation of genetic algorithm, it is hypothesized that when the number of vertices is reduced by $\mathrm{n} \%$, the computational time should be proportionally reduced to $(\mathrm{n} \%) 3$. The ratio of separated optimisation to whole structure optimisation in nodes, computation time \& converge time, and the fitness improvement for three tested structures are summarized in Table I. The fitness improvement is computed as $\left(F i t_{\text {sep }}-F i t_{\text {whole }}\right) / F i t_{\text {whole }}$.

TABLE I: SUMMARY OF EXPERIMENTAL RESULTS

\begin{tabular}{lccc}
\hline \hline & $\begin{array}{l}\text { Low } \\
\text { density }\end{array}$ & High density \\
\hline & & & \\
& & & \\
Clustered structure & 181 & 830 & 1351 \\
$\begin{array}{l}\text { Member size } \\
\text { Ratio of nodes } \\
\text { (upper: lower) }\end{array}$ & $1.3: 1$ & $1.5: 1$ & $2.7: 1$ \\
$\begin{array}{l}\text { Ratio of required time } \\
\text { (theoretical) }\end{array}$ & $25.5 \%$ & $28 \%$ & $41 \%$ \\
$\begin{array}{l}\text { Ratio of converge time } \\
\text { Fitness improvement }\end{array}$ & $152 \%$ & $35 \%$ & $61 \%$ \\
\hline \hline
\end{tabular}

Although with various ratios of nodes, the spectral clustering separates the space frame structures with minimal damage cutting at a reasonable plane or hyperplane. Different level of improvement in computational efficiency is observed by separating the clusters with high complexity, but the 
clustering algorithm can do more harm than good to the optimisation process when a structure has a low level of complexity. In general, spectral clustering can segment the space frame structures, but different structural geometries and different complexity level can pose an influence on the algorithm performance.

\section{A. Computational Time}

The computational time required for separated optimisation to reach convergence is highly reduced and is approximately proportional to the partition of the nodes, but the algorithm does not show its superiority in computational efficiency unless the sufficient number of nodes are involved in the optimisation process.

In the structure designed to have obvious substructures, a greater improvement in computational time is observed when the complexity is high. The reductions in converge time is smaller than the theoretical hypothesis, which might be due to the oversimplification of calculation of the big $\mathrm{O}$ notation of GA and varied CPU conditions. For the structure with lower complexity in the same shape, not only a longer time is required to converge, but also a worse ultimate fitness is observed. This phenomenon is preliminarily explained that when the number of nodes is efficiently small, FEA does not play a dominant part in the execution time, and the clustering makes it harder to find an appropriate ratio between the two substructures. So, when complexity is lower than a certain level, the addition of clustering process will lose its advantage.

The arbitrarily shaped structure without obvious clusters also presents a smaller time reduction than hypothesized. This gap is preliminary explained that this kind of structure is not a fully nearly-independent system. Because 30 connecting members between two substructures are cut by the spectral clustering, the intra-structure connections of the system are not sufficiently small compared with the connections within the substructure (Belkin and Niyogi [16]). Thus, the influence of breaking down the system is no longer negligible, and a large reduction in overall time is not guaranteed.

\section{B. Computational Efficiency}

Experimental results show that separating a space frame for structural optimisation always gain efficiency during the first several runs compared with the whole structure optimisation, but the subsequent improvement varies regarding structures' geometry properties such as topology and complexity. For structures beyond a certain level of complexity, the efficiency for segmented optimisation is around ten times higher than that of the whole-structure optimisation in the several initial runs, leading to a quick convergence. This considerable increase in efficiency attribute to the considerable reduction of the search space of GA, which speeds up the search process and allows the optimal solution to be found with a small number of iterations.

However, the oscillations in the clustered optimisation are observed to be much fiercer, regardless of load condition. This is demonstrated mostly in the structure with low-level complexity and the unevenly separated structures. The fluctuation is too strong to reach or hold an optimal fitness value, causing an equivalent or lower fitness value compared with the whole structural deflection. This instability possibly because FEA considers a structure as an interconnected whole, but it fails to examine the structure as a whole when optimising separately; thus, when the two substructures are combined back into the original configuration, the topology of the structure, the stiffness matrix and the nodal reaction will change, and the optimised radii in the segmented structure cannot adapt to the new load condition of the whole structure. In this case, the solution for separated optimisation can be inaccurate, and the scaling factor to rationalize the relative radii between the two subclusters plays a more critical role for structural stability. The scaling coefficient should be chosen more carefully and recalculated more frequently, especially for the structures whose connecting members play an unignorable role in the topology.

Considering the observations above, it can be presumed that to achieve a better solution. The clustered optimisation can be executed for 10 to 20 generations until it reaches the optimal solution. The whole-structure optimisation could then be used for the remainder of the search to achieve a further increase without considerable fluctuations.

\section{CONCLUSION}

The optimisation of irregular structures with high complexity lacks computational lightness, and the time required rises exponentially as the input data size increases. This project proposed a modified structural optimisation approach to improve computational efficiency. The spectral clustering algorithm is used to partition the structure into substructures to break down the problem size into two halves, and consequently, the search space of the optimisation algorithm is reduced to half. Genetic algorithm is then used to find the combination of the member sizes with which the structure has the least overall deflections. Experiments are conducted under different load conditions and with different structural geometries and densities.

The results verified the hypothesis that for a heterogeneous structure with high level of complexity, the use of spectral algorithm will lead to a significant efficiency improvement while providing an equivalent or better optimisation solution. The reduction in the computational time is a function of GA's search space, represented by the partition ratio of input vertices, and the magnitude of reduction varies according to the topology and the density of the structure.

The results are limited to specific load condition and structural geometries, composed mostly of vertically segmented shapes. Further investigations need to be done to examine the universal feasibility of this modified optimisation algorithm, including experiments with different numbers of clusters, various types of geometries and complex load conditions such as point load and dynamic load. Besides, a weighted similarity graph according to the member size can be used in the clustering algorithm to improve the accuracy of the partitioning, and a multi-objective genetic algorithm which optimizes not only the cross-sectional area, but also the topology and configuration are recommended to extend the range of applications of spectral clustering and meet practical needs.

\section{CONFLICT OF INTEREST}

The authors declare no conflict of interest. 


\section{AUTHOR CONTRIBUTIONS}

$\mathrm{S}$ Hanna provided the directional and technical support; $\mathrm{X}$ Zhuang conducted the research; X Zhuang and S Hanna analyzed the data; X Zhuang wrote the paper; all authors had approved the final version.

\section{REFERENCES}

[1] S. M. Shrestha and J. Ghaboussi, "Evolution of optimum structural shapes using genetic algorithm," J. Struct. Eng, vol. 124, issue 11, pp. 1331-1338, 1998.

[2] D. Mange and M. Tomassin, Bio-Inspired Computing Machines, Towards Novel Computational Architectures, 1st ed. Swiss: PPUR, 1998, ch. 4.

[3] B. Rylander and J. A. Foster, "GA Hard Problems," in Proc. GECCO, 2000, vol. 367, pp. 367-374.

[4] H. A. Simon, The Sciences of the Artificial, USA: MIT press. 1996, ch. 8.

[5] H. A. Simon, "The architecture of complexity: Hierarchic systems," The Sciences of the Artificial, MIT Press, pp. 183-216, 1996.

[6] M. Pelikan and F. G. Lobo, "Parameter-less genetic algorithm: A worst-case time and space complexity analysis," in Proc. GECCO, 2000, pp. 370-382.

[7] J. He J and X. Yao, "Drift analysis and average time complexity of evolutionary algorithms," Artif. Intell, vol. 127, no. 1, pp. 57-85, 2001.

[8] G. Rudolph, "How mutation and selection solve long path problems polynomial expected time," Evol. Comput, vol. 4, no. 2, pp. 195-205, 1997.

[9] I. Farmaga, P. Shmigelskyi, P. Spiewak, and L. Ciupinski, "Evaluation of computational complexity of finite element analysis," in Proc. IEEE CADSM, 2011, pp. 213-214.

[10] U. V. Luxburg, “A tutorial on spectral clustering," Stat. Comput, vol. 17, no. 4, pp. 395-416, 2007.

[11] F. Queyroi, "Optimizing a hierarchical community structure of a complex network," Advances in Knowledge Discovery and Management, vol. 527, pp. 3-14, 2014.

[12] J. Shi and J. Malik, "Normalized cuts and image segmentation," IEEE TPAMI, vol. 22, no. 8, pp. 888-905, 2000.

[13] A. Y. Ng, M. I. Jordan and Y. Weiss, "On spectral clustering: Analysis and an algorithm," AIPS, pp. 849-856, 2002.
[14] S. Hanna, "A representational scheme for the extraction of urban genotypes," in Proc. 5th DCC'12., 2012, pp. 471-488.

[15] D. Xu and Y. Tian, "A comprehensive survey of clustering algorithms," AODS, vol. 2, no. 2, pp. 165-193, 2015.

[16] M. Belkin and P. Niyogi, "Laplacian eigenmaps for dimensionality reduction and data representation," Neural Comput., vol. 15, no. 6, pp. 1373-1396, 2003.

Copyright (C) 2020 by the authors. This is an open access article distributed under the Creative Commons Attribution License which permits unrestricted use, distribution, and reproduction in any medium, provided the original work is properly cited (CC BY 4.0).

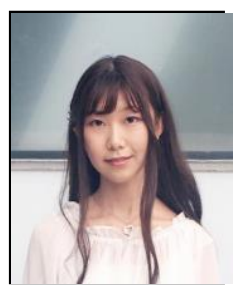

Xinwei Zhuang was born in China on Dec. 27, 1993 She received first honor degree in master of science, architectural computation from the Bartlett School of Architecture at University College London, London, UK in 2017.

She is a research assistant and architect. Her research interests lie in morphogenetic design, algorithm aided design, machine learning and geometric optimisation.

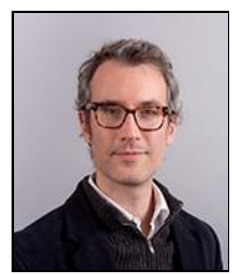

Sean Hanna is a reader in space and adaptive architectures at UCL, director of the Bartlett Faculty of the Built Environment's MSc/MRes programs in Adaptive architecture and computation, and academic director of UCL's EngD Centre for Virtual Environments, Imaging and Visualization.

His research is primarily in developing computational methods for dealing with complexity in design and the built environment, including the comparative modelling of space, and the use of machine learning and optimisation techniques for the design and fabrication of structures.

Dr. Hanna is a member of the UCL Space Group, noted as one of the UK's highest performing research groups in the field of architecture and the built environment in the last two consecutive UK Research Assessment Exercises. 